\title{
On the Performance of Compressed Sensing-based Methods for Millimeter-wave Holographic Imaging
}

\author{
Qiao Cheng ${ }^{1}$, Akram Alomainy ${ }^{1}$, And YANG HaO ${ }^{1, *}$ \\ ${ }^{1}$ School of Electronic Engineering and Computer Science, Queen Mary University of London, London E1 4NS, United Kingdom \\ *Corresponding author: y.hao@qmul.ac.uk
}

Compiled October 20, 2015

This paper investigates compressed sensing (CS) based methods for reducing data acquisition time in 2-dimensional (2-D) millimeter-wave holographic imaging systems. Specific attention is paid to situations where the array element spacing does not satisfy Nyquist criterion due to physical limitations. Both simulation and experimental results demonstrate that CS methods achieve better reconstruction than the conventional backpropagation method with undersampled data at the cost of increased computational complexity. Specifically, the definition based CS (D-CS) method derived by discretizing the scattering model achieves the best image resolution but can produce ghost targets when the sampling interval is greater than approximately twice the Nyquist sampling interval. On the contrary, the Fourier transform based CS (FT-CS) method has a relatively lower resolution but performs well in the case of low number of measurements, large sampling interval and low transmit power. In addition, the D-CS method requires much higher time complexity and space complexity than the FT-CS method since the 2-D data needs to be processed in vector form. Particularly, the space complexity of constructing and loading the dictionary matrix makes the D-CS method extremely inefficient in dealing with real time applications. The overall algorithm running time of D-CS method can be as high as 50 times of FT-CS method with a $81 \times 81$ scanning aperture and $121 \times 121$ grid size in reconstruction. An efficient way is to use the FT-CS method for coarse imaging and then use the D-CS method for specific regions where better precision is required. () 2015 Optical Society of America

OCIS codes: (090.1995) Digital holography; (110.1220) Apertures; (110.2960) Image analysis; (110.3010) Image reconstruction techniques.

http://dx.doi.org/10.1364/ao.XX.XXXXXX

\section{INTRODUCTION}

In the field of security scanning and nondestructive testing, Millimeter-wave (MMW) technology has recently gained substantial increase in popularity due to its high resolution and ability to penetrate common obstructions for imaging concealed weapons and other objects [1-3]. Among many MMW imaging techniques, holographic imaging is usually adopted for its better resolution and larger aperture size. MMW holographic imaging is an active imaging technique that operates by sampling the amplitude and phase of backscattered field from a given scene. The sampled hologram is then used for reconstruction with Fourier based backpropagation (BP) algorithm. However, due to the relative small wavelength, generating highresolution images using Fourier based techniques requires data acquisition over a uniformly and densely sampled set of points [3]. For a single transceiver system, the long collection time may be prohibitively costly. For example, it takes approximately
2 hours to mechanically scan an area of $400 \mathrm{~mm} \times 400 \mathrm{~mm}$ at $2 \mathrm{~mm}$ spacing using uniform raster scanning. To overcome this challenge, one would consider more sparsely sampled data collection strategies where the transceiver only samples at a small fraction of positions on the uniform grid. For instance, undersampling with certain trajectory is often adopted in magnetic resonance imaging (MRI) to save scan duration [4]. However, when inverse Fourier transform (IFT) is applied to sparsely sampled data with zero-filling, reconstruction quality can be poor (e.g. aliasing artifacts, low spatial resolution, reduced SNR).

The emerging field of Compressed Sensing (CS) $[5,6]$ has offered great insight into how to solve this issue. CS theory guarantees that sparse or compressible signals can be recovered from far fewer measurements than those were traditionally thought necessary. To date, this technique has been successfully applied to a wide range of imaging applications including; terahertz imaging [7-9], MMW imaging [10-13], synthetic aperture radar (SAR) imaging [14-22], medical imaging [23, 24] and in- 
verse scattering [25-27]. Specifically, in [12], CS was first introduced to single frequency MMW indirect holographic imaging for 2-D spatial undersampling. Fourier transform (FT) based forward and backward operators were adopted in the CS framework and 3D object estimation from 2-D measurements was realized by applying total variation (TV) regularization. The same strategy was then extended to 2-D direct MMW holography where both the amplitude and phase are acquired for image reconstruction [13]. Three sparsity bases, TV, wavelet and curvelet were tested for comparison. In [20-22], single frequency 2-D forward and backward operators have been extended to multi-frequency 3-D version for three-dimensional wideband SAR imaging. All these methods rely on existing FT model of traditional BP method, thus are referred to as Fourier transform based CS (FT-CS) method for simplicity.

Meanwhile, many CS methods are directly derived from the imaging scattering model by reformulating the model into a matrix form. In [15], CS was introduced to stepped frequency ground penetrating radars (GPSs) for compressed data acquisition both in frequency dimension and spatial dimension. The same idea was later applied to 2-D SAR imaging in [16] and [17]. Since this definition based CS (D-CS) method is a straightforward expansion of the scattering model, it can be easily implemented for different scanning apertures, e.g., planar aperture, circular aperture and cylindrical aperture. However, the main drawback is the high computational complexity since 2-D data has to be vectorized before reconstruction. In [18], a low complexity 3-D imaging method was presented. The complexity reduction is based on small bandwidth and narrow-angle measurement assumption which makes the multi-dimensional scattered data becomes separable and thus can be processed with Kronecker CS [19]. Another attempt to reduce complexity was made in [28] for multiple frequency 2-D imaging. A near-field approximation of the distance from antenna to each scattering center was proposed to make the angularly sampled data separable for 2-D FFT operation. Since both two methods rely on single transceiver to scan with very small angle interval and frequency interval, their use in other scanning apertures are quite limited.

Due to the enormous advances made in semiconductor technology over the last few years, highly integrated circuits with moderate costs are achievable in MMW frequencies [29, 30]. A practical imaging system for security scanning will undoubtedly adopt antenna array over single transceiver for the remarkably reduced scanning duration. Although many D-CS methods claim much better efficiency than traditional Fourier based method due to their random undersampling feature, this may not be the case for antenna array imaging systems when compared to FT-CS methods. Applying CS to array imaging systems is very different from applying CS to single transceiver systems. Firstly, to realize an antenna array such that the undersampling pattern can be completely arbitrary is not practical. On the contrary, the undersampling pattern should be designed according to the physical array aperture. Secondly, CS methods enables sub-Nyquist sampling by randomly undersampling the locations on a full grid that satisfy Nyquist criterion. However, due to many physical limitations, the engineering realization of such an antenna array is quite challenging. Commonly, a tradeoff is to increase the array element spacing, which is to say the full sampled data already violates Nyquist criterion.

The objective of this paper is to find an efficient method for near field MMW holographic imaging in terms of less imaging time and better image quality. To the authors' knowledge,

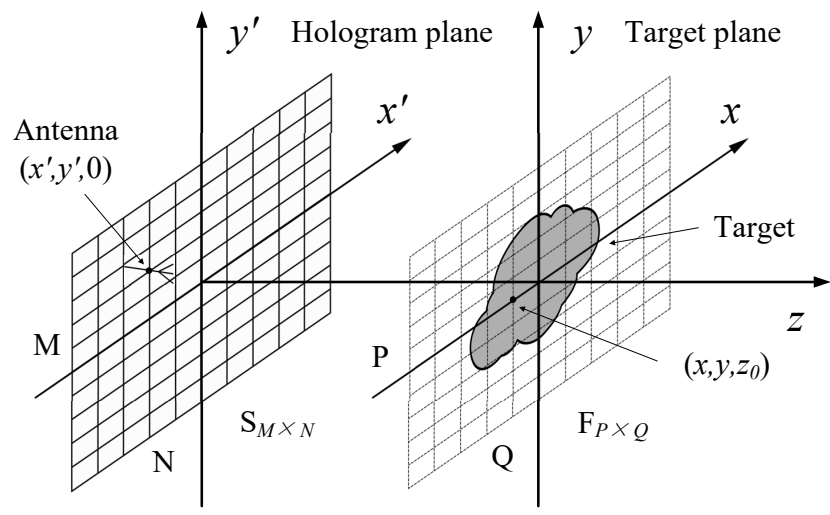

Fig. 1. Holographic imaging geometry.

D-CS method that has been extensively studied for wideband 2-D imaging and 3-D imaging is rarely mentioned in single frequency 2-D holographic imaging and its performance compared to FT-CS method is still unknown, especially in the aforementioned practical situations. More importantly, many papers only give simple comparison between CS methods and BP method with qualitative results. Therefore, we derive the D-CS method for the single frequency case and compare it with the FT-CS method and the traditional BP method in terms of image quality and computational complexity under various scenarios. Both the qualitative and quantitative results demonstrate that FT-CS method has higher robustness in the case of low number of measurements and large sampling interval. DCS method achieves better resolution with fine grid size setting but requires more measurements for stable reconstruction and extremely higher computational complexity.

The remainder of this paper is organized as follows. In section 2, we briefly introduce the theoretical background of single frequency 2-D holographic imaging. In section 3, two CS based approaches have been presented and analyzed. Also, we discussed the choices of sparsity constraints for reconstruction algorithm with numerical results. Section 4 gives the performance evaluation of different methods in terms of reconstruction quality and computational complexity.

\section{SINGLE FREQUENCY 2-D HOLOGRAPHIC IMAGING}

\section{A. Theoretical Background}

The configuration of an example imaging system is shown in Fig. 1. Here, we give the formulation of 2-D cross-range imaging based on single frequency. During the imaging process, a transceiver is scanned over a 2-D planar aperture (hologram plane) to measure signals reflected from the target plane. It is assumed that the transceiver is at position $\left(x^{\prime}, y^{\prime}, 0\right)$ and a general point $\left(x, y, z_{0}\right)$ is at the target plane. The reflectivity function of the target plane is assumed to be characterized by $f\left(x, y, z_{0}\right)$. Under Born approximation and ignoring the amplitude attenuation, the scattering field at the transceiver can be approximately represented by a linear superposition of reflected waves from each point on the target plane or

$$
s\left(x^{\prime}, y^{\prime}\right)=\iint f(x, y) \exp (-j 2 k R) d x d y,
$$

where $R=\left[\left(x-x^{\prime}\right)^{2}+\left(y-y^{\prime}\right)^{2}+\left(z_{0}-0\right)^{2}\right]^{1 / 2}, k=\omega / c$ is the wavenumber, $\omega$ is the angular frequency and $c$ is the speed 
of light. The most common technique to solve Eq. (1) is BP algorithm [31] which was originally developed from acoustic holography based on angular spectrum decomposition [32]. BP algorithm can be simply summarized as

$$
f(x, y)=F T_{2 D}^{-1}\left[F T_{2 D}[s(x, y)] \exp \left(-j k_{z} z_{0}\right)\right],
$$

where $k_{x}, k_{y}$ and $k_{z}=\left(4 k^{2}-k_{x}^{2}-k_{y}^{2}\right)^{1 / 2}$, according to the dispersion relation, are wavenumbers in $x, y$, and $z$ dimension, respectively. $F_{2 D}\{\cdot\}$ and $F T_{2 D}^{-1}\{\cdot\}$ represent 2-D FT operator and its inverse, respectively. The final image of the target can be acquired by computing the amplitude of the complex valued reflectivity function $f$. The exponential term in Eq. (2) compensates for wavefront curvature of the electromagnetic wave and thus makes this algorithm suitable for near-field imaging.

It should be noted that there exists other similar algorithms like frequency-wavenumber (F-K) migration [33], from geophysics community and, SAR [34], from the radar community. All these algorithms are actually a simplification of the complicated inverse scattering problem derived from the Maxwell's equations. Interested readers are referred to [35] for a detailed discussion of these algorithms in the inverse scattering point of view.

\section{B. Nyquist Sampling Interval}

To avoid aliasing in image reconstruction, the sampling interval has to satisfy Nyquist theorem during the acquisition process. This sampling interval is determined by a number of factors including the wavelength $\lambda$, antenna beamwidth $\theta_{a}$, aperture size $D$, target size and distance to the target $R$. Although there is no good criterion so far with strict mathematical formulation considering these physical parameters for near field imaging systems, Joseph et al. [36] has suggested that the optimal sampling interval should be smaller than the theoretical resolution in order to achieve it. The theoretical cross-range resolution can be expressed as

$$
\delta \approx \frac{\lambda}{4 \sin (\theta / 2)} \mid \theta=\min \left(\theta_{a}, \theta_{b}\right)
$$

where $\theta_{b}=2 \arctan (D / 2 R)$ is the angle subtended by the scanning aperture.

It has been shown in [36], resolution $\delta$ and also the Nyquist sampling interval are inversely proportional to the distance $R$. The most restrictive case where the target is very close to the aperture requires the sampling interval to be on the order of $\lambda / 4$. For a practical imaging system that usually has a moderate transceiver-to-target distance, $\lambda / 2$ sampling interval is sufficient [37].

\section{COMPRESSED SENSING BASED APPROACHES}

Conventional imaging techniques must follow the Nyquist sampling theorem to avoid image aliasing. CS based approaches, on the other hand, enable image reconstruction from far fewer measurements by just randomly measuring a small fraction of positions on the scanning aperture. In this section, we first formulate the D-CS method for single frequency 2-D planar aperture case, then we give the matrix version of FT-CS method for the same set-up. Followed by a discussion of the effect of sparsity constraint to the complex-valued data.

\section{A. D-CS Model}

D-CS model can be directly derived from the signal model in Eq. (1) by discretizing the target plane into a grid of point scatters and then reshape the 2-D hologram matrix and reflectivity function matrix into long 1-D column vectors. Therefore, the 2-D imaging problem can be solved with standard 1-D CS reconstruction algorithms. Suppose the target plane reflectivity function $\boldsymbol{F}$ is discretized as a $P \times Q$ matrix and the hologram matrix $S$ has $M \times N$ sampling positions, as shown in Fig. 1. According to Eq. (1), a discrete version of $s(x, y)$ at $(m, n)$ of $S$ can be represented as

$$
s(m, n)=\sum_{i=1}^{P} \sum_{j=1}^{Q} f(i, j) \exp (-j 2 k R(m, n, i, j)),
$$

where $R(m, n, i, j)$ is the distance between the two positions located at $(m, n)$ of $S$ and $(i, j)$ of $\boldsymbol{F}$, respectively. By vectorizing $S$ and $F$, e.g., concatenating the columns of a matrix into a single column, Eq. (4) can be reformulated as

$$
\begin{gathered}
\boldsymbol{s}=\boldsymbol{A} \boldsymbol{f} \\
{\left[\begin{array}{c}
s(1,1) \\
\vdots \\
s(M, 1) \\
\vdots \\
s(1, N) \\
\vdots \\
s(M, N)
\end{array}\right]=\left[\begin{array}{c}
\boldsymbol{a}(1,1)^{T} \\
\vdots \\
\boldsymbol{a}(M, 1)^{T} \\
\vdots \\
\boldsymbol{a}(1, N)^{T} \\
\vdots \\
\boldsymbol{a}(M, N)^{T}
\end{array}\right]\left[\begin{array}{c}
g(1,1) \\
\vdots \\
g(1,1) \\
g(P, Q) \\
\vdots \\
g(P)
\end{array}\right.}
\end{gathered}
$$

where

$$
\begin{gathered}
\boldsymbol{a}(m, n)=[\exp (-j 2 k R(m, n, 1,1)), \ldots, \exp (-j 2 k R(m, n, P, 1) \\
), \ldots, \exp (-j 2 k R(m, n, 1, Q)), \ldots, \exp (-j 2 k R(m, n, P, Q))]^{T},
\end{gathered}
$$

and $A$ can be seen as a dictionary matrix. Suppose we are using a single transceiver system, the total number of measurements can be reduced by programming the transceiver to randomly sampling a fraction of all the positions on the grid. Mathematically, this amounts to introducing a binary mask as

$$
Y=M . * S,
$$

where $* *$ represents element-wise multiplication, $Y$ is the undersampled hologram and $S$ is the fully sampled hologram. Binary mask $\boldsymbol{M}$ is an $M \times N$ sampling matrix which only contains 1 (sample) and 0 (ignore). Suppose the number of sampled points is $K$, then the sampling rate can be computed as the ratio of $K$ to $M N$. Before applying reconstruction, $M$ needs to be transformed to fit vectorized $S$. We denote by $\dot{M}$ the transformed version of $\boldsymbol{M}$. Then the vectorized undersampling process can be summarized as

$$
y=\dot{M} s=\dot{M} A f,
$$

where $y$ is the $K \times 1$ vector representation of $\boldsymbol{Y}$ and $\dot{M}$ is a $K \times$ $M N$ matrix.

The resolution of the reconstructed image is directly related to the discretization of the target plane. Clearly, finer grid size will provide higher resolution. However, this operation leads to 
significantly increased size of $A$ and thus introduce higher computational complexity. This problem gets more serious when dealing with $3-\mathrm{D}(x$-dimension, $y$-dimension and frequency dimension) data. The complexity reduction methods that based on small angular scanning aperture with different approximations of the scattered data $[28,38]$ become invalid here since in the case of planar scanning aperture, the square root phase term cannot be easily separated.

In addition to the increased complexity, finer grids will also weaken the performance of CS reconstruction. Firstly, as can be seen from Eq. (5), the only difference among the elements in $A$ is the distance $R$, which means the closer the adjacent points on the grid, the more similarities among the columns in $A$. In the CS framework, more similar columns means higher mutual coherence [39], which should always be avoid. Secondly, finer grids will increase the number of unknowns and thus require more measurements for stable CS reconstruction.

\section{B. FT-CS Model}

FT-CS model is based on the conventional BP algorithm that takes advantage of FFT. Similar to D-CS model, all data for FTCS model must be vectorized before applying recovery algorithms. Such vectorization process introduce unnecessary complexity and memory requirement in algorithm deployment. Actually, with the knowledge of forward operator and backward operator, we can directly use matrix formulation in computation if gradient descent based recovery algorithms are adopted.

Based on the backward process in Eq. (2), the forward process can be written as

$$
s(x, y)=F T_{2 D}^{-1}\left[F T_{2 D}[f(x, y)] \exp \left(j k_{z} z_{0}\right)\right] .
$$

To better describe the FT-CS method, we denote forward process in Eq. (8) as

$$
S=H F,
$$

where $\boldsymbol{H}=F T_{2 D}^{-1}\left[F T_{2 D}[\cdot] \exp \left(j k_{z} z_{0}\right)\right]$ is an operator. Similarly, the adjoint system model of Eq. (9) (backward process) is

$$
F=H^{\dagger} S,
$$

where $\boldsymbol{H}^{\boldsymbol{\dagger}}=F T_{2 D}^{-1}\left[F T_{2 D}[\cdot] \exp \left(-j k_{z} z_{0}\right)\right]$. Therefore, by adding the undersampling mask, the FT-CS model can be written as

$$
Y=M . * S=M . * H F,
$$

\section{Reconstruction Algorithm}

There are many CS algorithms available to solve the underdetermined system in Eq. (7) and Eq. (11). Greedy pursuit [40] and $\ell_{1}$ optimization [6] are the two mainstreams. In comparison, greedy algorithms are generally much faster but require more measurements. However, to solve FT-CS model with greedy algorithms, the forward and backward linear operators involving 2D FT and IFT should be transformed into matrix forms, which requires too much computation and makes the problem more complicated. Here, we adopt the two-step iterative shrinkagethresholding (TwIST) algorithm [41] which belongs to the $\ell_{1}$ optimization category. TwIST is a variant of iterative shrinkagethresholding (IHT) and is significantly faster than traditional $\ell_{1}$ algorithms like Basis Pursuit [42]. Moreover, the forward and backward operators can be applied directly without any modification.

One of the key requirements for successful CS reconstruction is the image sparsity or compressibility. Natural images are compressible by sparse representation such as those from a

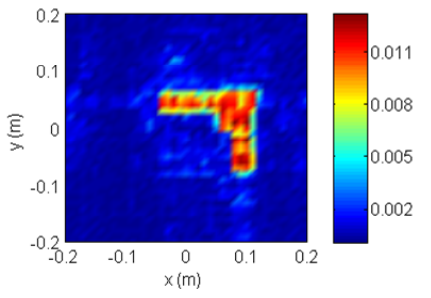

(a)

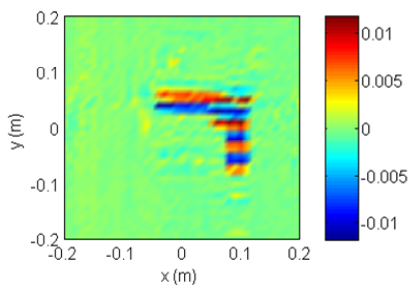

(c)

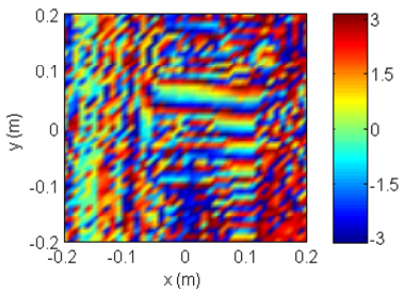

(b)

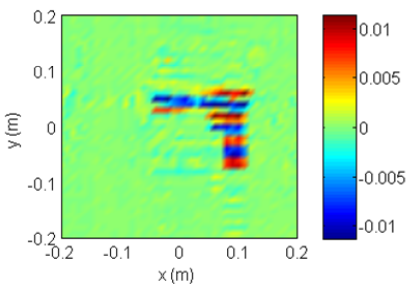

(d)
Fig. 2. The (a) Amplitude, (b) phase, (c) real and (d) imaginary part of the reconstructed image by BP method.

wavelet transform and discrete cosine transform (DCT). In our case of study, target recognition is the first priority. Therefore, total variation (TV) regularization [43] can be adopted since it can preserve the sharp edges of an image while imposing smoothness on the solution. TV refers to the integral of the absolute gradient of the signal. The discrete version for a 2-D image $F$ can be defined as

$$
\operatorname{TV}(\boldsymbol{F})=\sum_{i, j} \sqrt{\left|\boldsymbol{F}_{i+1, j}-\boldsymbol{F}_{i, j}\right|^{2}+\left|\boldsymbol{F}_{i, j+1}-\boldsymbol{F}_{i, j}\right|^{2}}
$$

where $i$ and $j$ denote the discrete indices of $\boldsymbol{F}$. Then the corresponding optimization problem for Eq. (7) and Eq. (11) with TV regularization can be written as

$$
\hat{f}=\underset{f}{\operatorname{argmin}}\|\dot{M} A f-y\|_{2}+\alpha \operatorname{TV}(\boldsymbol{F})
$$

and

$$
\hat{\boldsymbol{F}}=\underset{\boldsymbol{F}}{\operatorname{argmin}}\|\boldsymbol{M} . * \boldsymbol{H} \boldsymbol{F}-\boldsymbol{Y}\|_{2}+\alpha \operatorname{TV}(\boldsymbol{F}),
$$

respectively. An important part of Eq. (13) and Eq. (14) is the selection of the regularization parameter $\alpha$, which controls the tradeoff between the sparsity of the solution and the closeness of the solution to the image. For example, if $\alpha$ is too small, then the solution is close to the conventional least squares solution, while if $\alpha$ is too large then the bias of the estimation will be very high. The optimal value of $\alpha$ is inconsistent across different data sets, and may change slightly for the same data set with different sampling rate.

As mentioned previously, the data in our direct holographic imaging system is complex-valued. Fig. 2 gives an example of the amplitude, phase, real and imaginary parts of a gun-shape target recovered by BP algorithm from experimental data. It is important to note that most sparsifying transforms for real-valued images become invalid or less effective for complex-valued data since only the amplitude of the image can be treated as sparse. Many CS based papers have ignored this fact by directly applying the sparsity constraint to the complex-valued signals or only use sparse point-like targets for simplicity. Nevertheless, there has been some attempts to solve this problem. In [44], the TV regularization is applied 


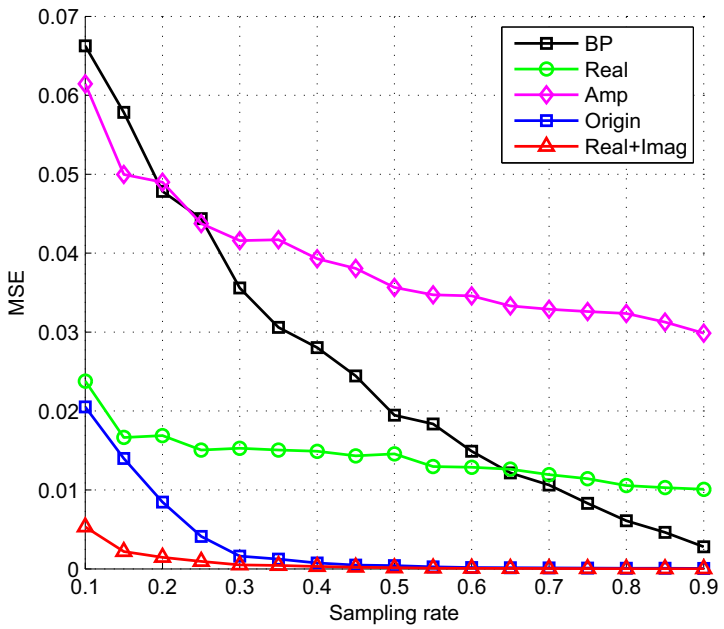

(a)

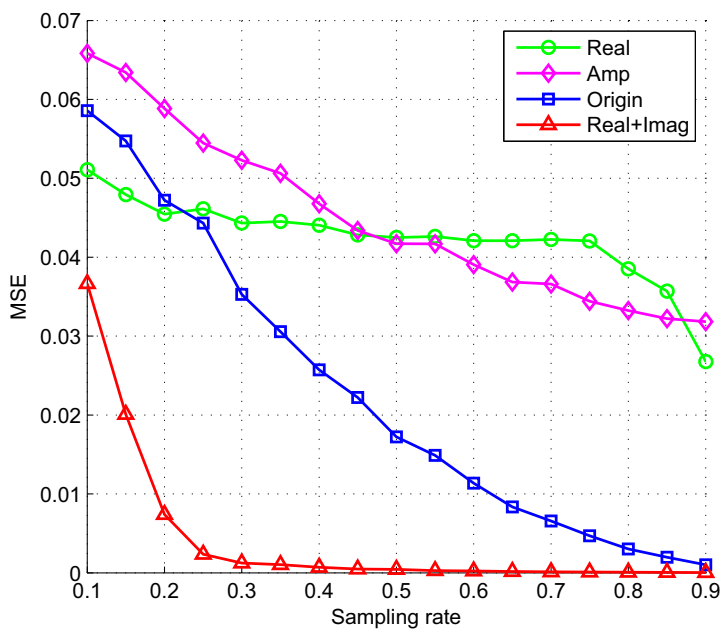

(b)

Fig. 3. MSE of reconstructed images of different sparsity constraints. (a) FT-CS and (b) D-CS.

to the amplitude only, but the phase is ignored, since in SAR images the phase is usually assumed to be random. On the contrary, the phase information for MRI and terahertz imaging is considered to be smooth and not vary rapidly. In [45], a phase smoothness constraint is introduced by controlling the similarities of the phase intensities among the image pixels. In [46], it was suggested that sparsity can be enforced separately in the real and imaginary parts. Both methods have shown to be effective in dealing with complex-valued image. In our case, the phase information of the acquired data can be seen as between random and smooth varying. How to find an optimal sparsity constraint shall be an object of further research.

For simplicity, we adopted TV regularization to both the real and imaginary data presented in this paper. Fig. 3 compares the proposed scheme to some other sparsity constraints by calculating their mean squared errors (MSEs) for the same experiment data as shown in Fig. 2. The MSEs are averaged over the results of 20 independent trials for each sampling rate. For FT-CS methods, the reconstructed image using full sampling rate with our scheme is used as reference image. Same configuration also applies to the D-CS method. The regularizations to the imaginary part only, phase part only and both amplitude and phase

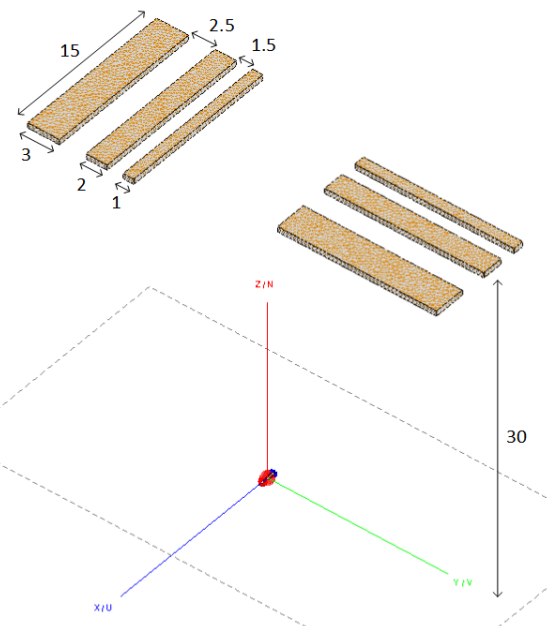

Fig. 4. Simulation model in FEKO. All dimensions are in $\mathrm{mm}$.

parts do not always converge thus are not shown here. As can be seen from Fig. 3, TV regularization to the amplitude only scheme (pink curve) actually does not perform well in our case. Moreover, our scheme (red curve) always achieves lower MSE than regularization applied directly to the original data scheme (blue curve) in both FT-CS and D-CS methods.

Finally, the modified optimization model with TV regularization to both real and imaginary parts for Eq. (13) and Eq. (14) can be written as

$$
\hat{\boldsymbol{f}}=\underset{f}{\operatorname{argmin}}\|\dot{\boldsymbol{M}} \boldsymbol{A} \boldsymbol{f}-\boldsymbol{y}\|_{2}+\alpha\left[\mathrm{TV}\left(\boldsymbol{F}_{\boldsymbol{r}}\right)+\mathrm{TV}\left(\boldsymbol{F}_{\boldsymbol{i}}\right)\right]
$$

and

$$
\hat{\boldsymbol{F}}=\underset{\boldsymbol{F}}{\operatorname{argmin}}\|\boldsymbol{M} . * \boldsymbol{H} \boldsymbol{F}-\boldsymbol{Y}\|_{2}+\alpha\left[\operatorname{TV}\left(\boldsymbol{F}_{\boldsymbol{r}}\right)+\operatorname{TV}\left(\boldsymbol{F}_{\boldsymbol{i}}\right)\right],
$$

respectively, where $\boldsymbol{F}=\boldsymbol{F}_{\boldsymbol{r}}+j \boldsymbol{F}_{\boldsymbol{i}}$.

\section{NUMERICAL ANALYSIS}

This section presents the performance analysis and comparison of two CS based methods using simulation results. The simulated data can be acquired using either simplified signal model in Eq. (1) (synthetic data) or full wave electromagnetic (EM) solver that follows rigorous Maxwell's equations. In many CS SAR imaging literature, point scatters are assumed and synthetic data is used for simplicity. However, in practice, the target area might be very complex and thus full wave simulation should be adopted to better approximate the real scenario/environment. We use FEKO EM software [47] to generate input data (measured hologram data) for the algorithms.

For fair comparison, we ensure that firstly all the methods use the same sampling pattern; secondly, the regularization parameter for both CS methods are adjusted appropriately so that the reconstructed images for all methods are optimized; and thirdly both CS methods share the same stopping criterion, that is the optimizations cease when the relative change in the objective function falls below $1 \times 10^{-4}$ or the number of iterations reached 10000. All the evaluations were implemented using Matlab 2014b (x64) on a Windows 7 operation system, with an Intel I7-4770 processor and 16 GB of memory.

The 3-D system geometry is illustrated in Fig. 4. An $x$-polarized dipole antenna working at $100 \mathrm{GHz}$ is used as 


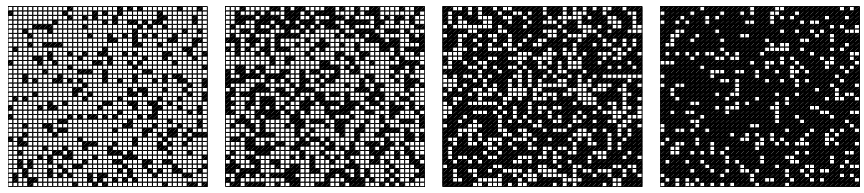

(a)

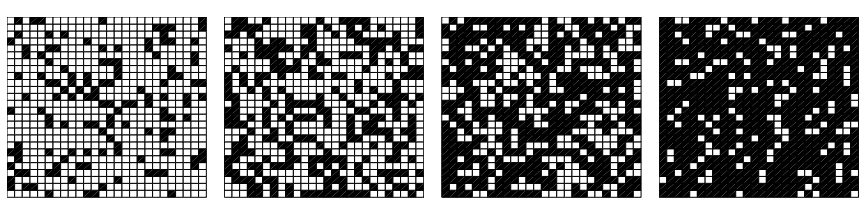

(b)

Fig. 5. Four random undersampling masks under (a) $\lambda / 2$ element spacing and (b) $3 \lambda / 4$ element spacing. From left to right, sampling rates of four masks are $80 \%, 60 \%, 40 \%$ and $20 \%$, respectively.

transceiver to scan an area of $60 \mathrm{~mm} \times 60 \mathrm{~mm}$. The target plane is parallel to the scanning aperture with a height of $30 \mathrm{~mm}$. Two identical targets which both consist of three metallic rectangular cuboids are placed perpendicularly to each other. The background medium is free space. During the simulation, the reflection scattering parameter (S11) of the antenna is acquired at each sampling position and is calibrated by subtracting the background $S 11$ as described in [48].

\section{A. CS with Sub-Nyquist Sampling Interval}

A practical implementation of concealed weapon detection system requires a scan in about 1-2 s which is only possible with electronically switched antenna array. However, physical considerations like antenna gain, beamwidth and mutual coupling between antennas make engineering realization of such an antenna array that satisfy Nyquist sampling interval very difficult in MMW frequency range. Generally, a tradeoff is to increase the antenna spacing, for example, $2 \lambda / 3$ [37] or even higher. Under such circumstances, if we use a linear array to perform 1D mechanical scanning and 1-D electronic scanning, then the Nyquist criterion can be satisfied only in the mechanic scanning dimension; if we use a rectangular array to perform 2-D electronic scanning, Nyquist criterion will be violated in both dimensions. In the first case, there is a trade-off between image quality and scan duration on how to choose the sampling rate in each dimension. Normally, undersampling in the mechanical scanning dimension can save more time since skipping one position means abandoning one line of measurements in the electronic scanning dimension. However, the highly undersampled data plus randomness reduced sampling pattern will greatly impair the reconstruction. In the second case, undersampling can be completely random in both dimensions. The already fast scanning time can be further reduced to enable real time imaging with CS methods. For the following discussions, we only consider the second case for simplicity.

\section{A.1. Qualitative Results}

Since the transceiver-to-target distance is $30 \mathrm{~mm}$, the Nyquist sampling interval is approximately $0.35 \lambda$ according to Eq. (3). Therefore, we acquired two sets of data with uniform undersampling interval (element spacing) of $\lambda / 2$ and $3 \lambda / 4$. These uniformly sampled data are then randomly sub-sampled by $80 \%, 60 \%, 40 \%$ and $20 \%$, as shown in Fig. 5, to simulate the random undersampling process. Here, the white pixels represent selected locations to sample. It can be noticed that since all cases have the same scanning region, small sampling interval case has denser pixels. Based on these sampling masks, conventional BP method and two CS based methods are employed for image reconstruction.

As mentioned previously, the gird size of the target plane has a crucial influence on the performance of D-CS reconstruction. Therefore, we also adopt three different grid spacings of $\lambda / 8, \lambda / 4$ and $\lambda / 3$ for D-CS method. It is important to note that due to the differences in size of reconstructed image and measurement matrix, two CS methods have different optimal regularization parameters. For instance, in the following example, the regularization parameter is around 0.01 for D-CS method and 0.001 for FT-CS method.

Fig. 6 shows the corresponding reconstructed images based on the two sets of undersampled data. As we can see from Fig. 6(a), different grid sizes lead to different image resolutions in reconstruction. The number of pixels of the three grid sizes are $161 \times 161,81 \times 81$ and $61 \times 61$, from left to right, respectively. It can be noticed that finer grid size does not actually give better image reconstruction, especially when the sampling rate is low. This agrees with our analysis in section 2 since the $\lambda / 2$ element spacing case is already highly undersampled compare to the Nyquist rate, reducing the grid size will require more measurements to mitigate effect of the increase of the mutual coherence, as well as the number of unknowns to be reconstructed. However, this does not mean that larger grid size always gives better results. Grid size should be carefully chosen because if it is too large that leads to very low resolution and grid mismatch [49] may also occur at this case. The optimal grid size should consider both the sampling rate and the array element spacing such that the number of measurements are well above the minimum requirement posed by the CS theory. On the contrary, the FT-CS method and BP method cannot freely choose the image size during reconstruction. This is because either 2-D FT or 2-D IFT of a matrix does not change its size, which makes the reconstructed image the same resolution as the sampled aperture. It should be noted that although some conventional smoothing techniques like zero-padding and interpolation can also be used within the FT-CS method, they do not really increase the spatial resolution, thus are not discussed here.

Fig. 6(b) shows the results based on $3 \lambda / 4$ element spacing which is about twice the Nyquist sampling interval. Compare to Fig. 6(a), FT-CS method and BP method have blurrier images since larger element spacing results in less pixels in the reconstruction. Interestingly, all D-CS methods with different grid sizes fail to reconstruct the image correctly as ghost targets can be observed in all cases. The FT-CS method, on the other hand, can still reconstruct the contour of the targets under very low sampling rate. This interesting result indicates that FT-CS method is much more robust to random undersampling than DCS method. Here, we believe FT and IFT in each iteration helps to retrieve the target information in the spatial domain.

\section{A.2. Quantitative Results}

While Fig. 6 only considers reconstructions with certain undersampling masks, Fig. 7 quantitatively summarizes the effect of random undersampling on the MSEs of reconstructed images of different methods. Since D-CS method fails to reconstruct images with $3 \lambda / 4$ element spacing, Fig. 7 only shows the result based on element spacing of $\lambda / 2$ and $\lambda / 4$. For the ease of annotation, we use H81 and I161 in the legend to indicate 


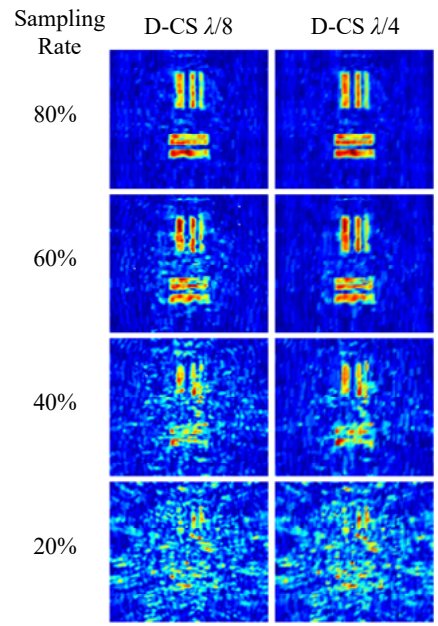

FT-CS

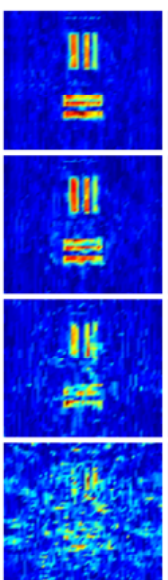

(a)
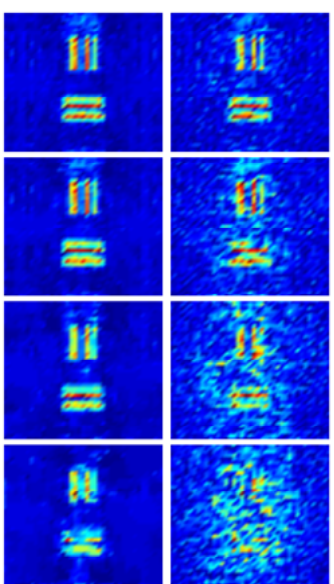

D-CS $\lambda / 4$

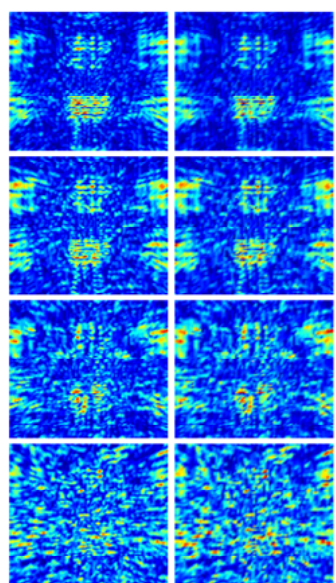

D-CS $\lambda / 3$

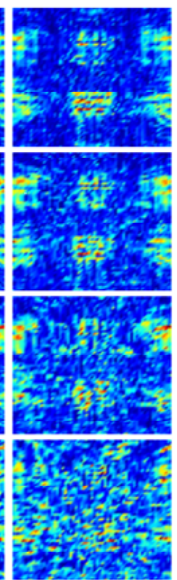

(b)

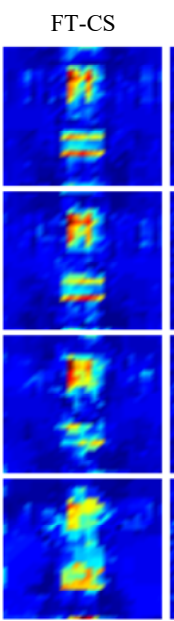

$\mathrm{BP}$

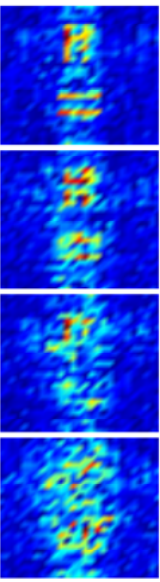

Fig. 6. Simulation results showing the reconstruction quality of three methods based on (a) $\lambda / 2$ element spacing and (b) $3 \lambda / 4$ element spacing. In each figure, from left to right, D-CS methods with grid size of $\lambda / 8, \lambda / 4$ and $\lambda / 3$, FT-CS method and BP method.

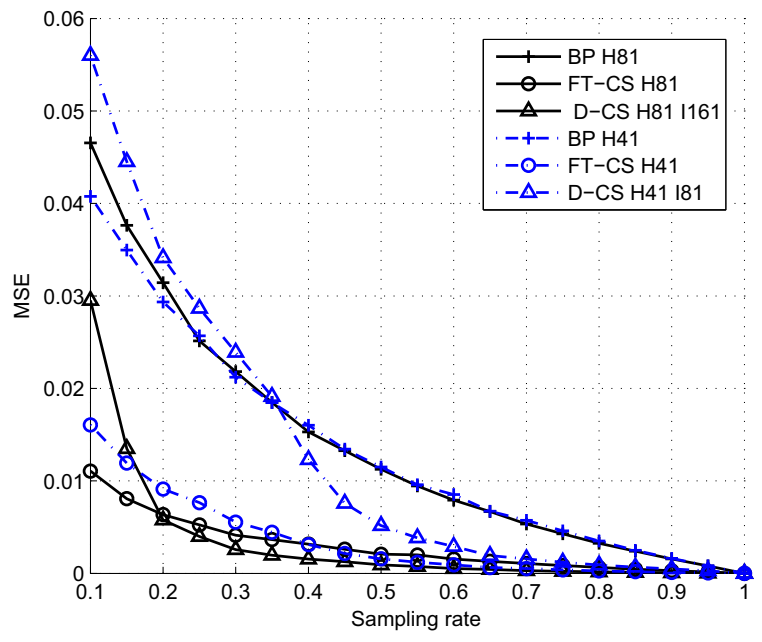

Fig. 7. MSE trend of three methods with element spacing of $\lambda / 2$ and $\lambda / 4$.

the hologram (scanning aperture) has $81 \times 81$ samples $(\lambda / 4$ element spacing) and the reconstructed image has $161 \times 161$ pixels ( $\lambda / 8$ grid spacing). The MSE of each method is calculated with respect to its own full sampling rate result and is averaged over the results of 40 independent trials for each sampling rate. An MSE curve with slow variation against sampling rate means the corresponding method is robust to random undersampling. Clearly, FT-CS achieves the best robustness in both element spacing cases which agrees with our qualitative results. More importantly, each method behaves distinctly in two element spacing cases. The MSE of D-CS method starts to vary intensively when the sampling rate is below $50 \%$ in $\lambda / 2$ element spacing case but a similar variation only happens under $20 \%$ sampling rate in the $\lambda / 4$ element spacing case. On the contrary, the BP method and FT-CS method have relatively small differences in the two cases. This indicates D-CS method is very susceptible to the element spacing or uniform undersampling interval. Increasing the number of measurements is recommended

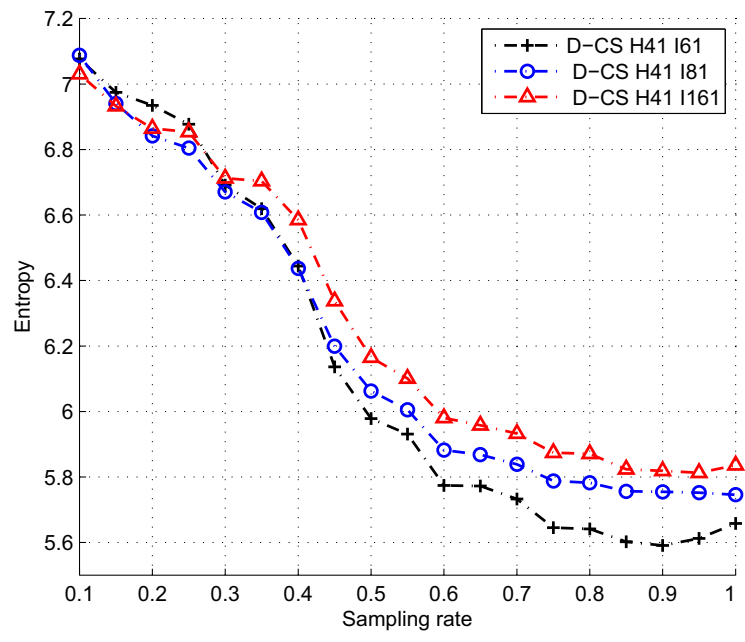

Fig. 8. Entropy of D-CS methods with gird size of $61 \times 61,81 \times$ 81 and $161 \times 161$.

for stable reconstruction.

However, it is not quite straightforward to compare the image quality among different methods since firstly, it is difficult to find an appropriate reference image for all methods with different element spacings and grid sizes, and secondly, different regularization parameters of CS algorithm result in distinct estimation biases and thus render the MSE metric ineffective to represent the true quality of the reconstruction. Hence, for simplicity, we only compare the image quality of D-CS method among different grid sizes. Minimum entropy is often used as a quality objective to focus image in SAR imaging algorithms [50] and thus is adopted here as the metric to approximately represent the image quality. As we can see from Fig. 8, the D-CS method with larger grid sizes achieves lower entropy, especially in the case of high sampling rate. When the sampling rate goes below $30 \%$, the randomness of three schemes start to dominate as also can be seen from Fig. 6 . 
Table 1. Complexity Comparison of D-CS Methods

\begin{tabular}{cccc}
\hline Configuration & Computing time (s) & Loading time (s) & Storage \\
\hline$M=41, N=81$ & 15.03 & 1.67 & $72 \mathrm{MB}$ \\
$M=41, N=121$ & 33.61 & 3.84 & $221 \mathrm{MB}$ \\
$M=81, N=121$ & 140.67 & 17.28 & $1.04 \mathrm{MB}$ \\
$M=81, N=161$ & 224.36 & 27.12 & $1.37 \mathrm{~GB}$ \\
$M=134, N=134$ & $1.45 \times 10^{3}$ & 88.65 & $2.94 \mathrm{~GB}$ \\
$M=134, N=178$ & $4.58 \times 10^{3}$ & 717.72 & $8.23 \mathrm{~GB}$ \\
\hline
\end{tabular}

\section{B. Computational Complexity}

Next, we consider the computational complexities of both CS methods. The BP method is the fastest among all three methods as CS based methods need multiple iterations until convergence. In general, the FT-CS method is more computationally efficient than the D-CS method due to the adoption of the FFT algorithm. Since both CS methods use the same reconstruction algorithm, we can compare the D-CS model and the FT-CS model for simplicity. Suppose the full scanning aperture is of size $M \times M$, the number of actually sampled points is $\mathrm{K}$, the discretization of D-CS method is $N \times N$. D-CS model in Eq. (7) consists of two matrix multiplication thus has time complexity of order $\mathcal{O}\left(K M^{2} N^{2}+K N^{2}\right)$. If we keep $M$ fixed, then the number of gird points $N$ contributes most to the time complexity while the number of sampled points $K$ has less contribution. For FTCS model in Eq. (11), it includes two 2-D FFT/IFFT operations and two element-wise matrix multiplication. Considering the $\mathcal{O}(M \log M)$ complexity of 1 -D FFT and $\mathcal{O}\left(M^{2}\right)$ complexity of element-wise matrix multiplication, the total time complexity is then about $\mathcal{O}\left(2 M^{2}+4 M^{2} \log M\right)$ for FT-CS method. Since the grid size of target plane is usually set to be finer than the sampling interval $(N>M)$, the D-CS method will always has much higher time complexity than the FT-CS method.

As the time complexity quantifies the amount of time that one algorithm needs to run, the space complexity is a measure of the amount of working storage an algorithm needs. The space complexity of D-CS method mainly comes from the dictionary matrix $A$ which contains the distance information from all pixels (voxels for 3-D case) in target area to all sampling points in the aperture plane. According to Eq. (5), an $M \times M$ scanning aperture with $N \times N$ grid points will lead to an $M^{2} \times N^{2}$ complex-valued $A$. Table 1 gives the computing time, loading time and required storage of $A$ based on different scanning apertures and grid sizes. Note the data processed in Matlab is set as double precision. While it is quite normal for any practical imaging system to deal with sampling aperture as large as $200 \times 200$, the D-CS method will be incredibly slow in such cases.

To verify the above analysis, we evaluate the overall algorithm running time of each method against the sampling rate as shown in Fig. 9. The test data has a $81 \times 81$ scanning aperture with $81 \times 81$ and $121 \times 121$ grid sizes adopted for the D-CS method. The running times are averaged over the results of 10 independent trials for each sampling rate. The dark colored curves show the superiority of FT based methods over D-CS methods. For example, the D-CS method with $N=121$ has a running time nearly 50 times of the FT-CS method when the sampling rate is $50 \%$. Further, the dashed curves give a better illustration of the FT-CS method and the BP method with a smaller scale. Both methods have little variation in running

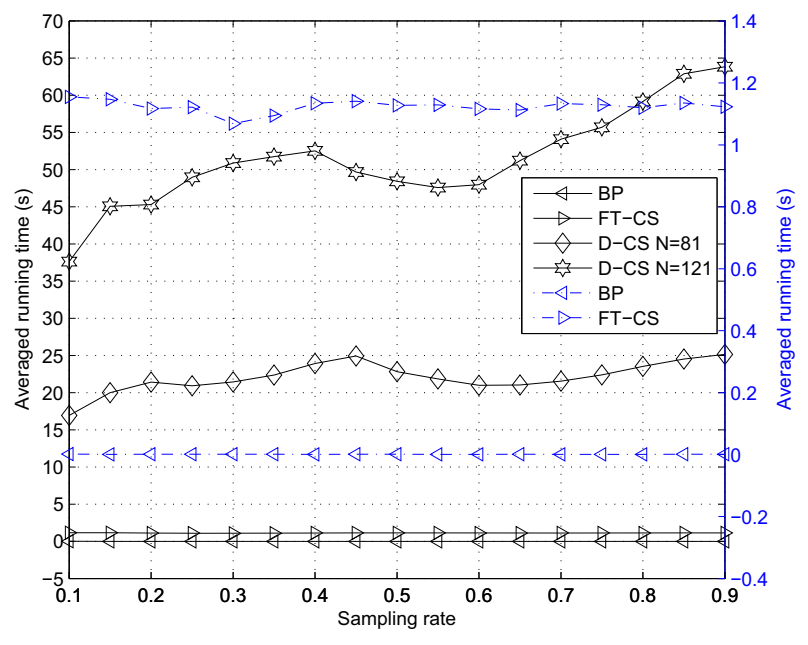

Fig. 9. Averaged running time of different methods versus random undersampling.

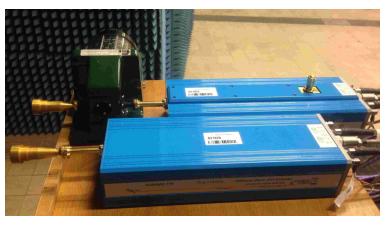

(a)

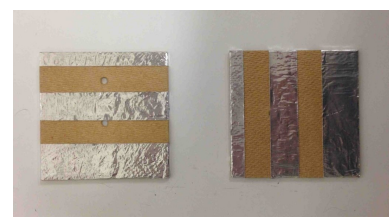

(b)
Fig. 10. System setup: (a) measuring equipments and (b) two identical targets with metallic stripes.

time as sampling rate increases. However, for the D-CS method, the variation becomes obvious when $N$ is bigger. This is expected as the number of grid points contributes more to the time complexity.

In summary, for the D-CS method, the constructing and loading time of the dictionary matrix $A$ is non-negligible since the target distance might be unknown in reality. The high memory requirement of this matrix will extremely slow down the D-CS reconstruction in real time calculation. An efficient way is to use the FT-CS method for coarse imaging and then use the DCS method for the specific small areas where higher resolution is required.

\section{EXPERIMENTAL RESULTS}

To validate the simulation, we conducted several experiments in an anechoic chamber. In Fig. 10(a), two corrugated conical horn antennas with frequency multipliers are connected to a vector network analyzer (VNA) to work as transmitter and receiver, respectively. Both antennas have a flare angle of $15^{\circ}$. The normal transmit power is between $5 \mathrm{dBm}$ and $10 \mathrm{dBm}$. Our targets are two wooden plates with metallic stripes as shown in Fig. 10(b), with a distance of $1.6 \mathrm{~m}$ to the scanning aperture. As it is not easy to do raster scan with the bulky frequency multipliers, the measuring equipments are kept still while the target is mounted on the scanner instead. The two targets are placed perpendicular to each other to scan an area of $400 \mathrm{~mm} \times 400 \mathrm{~mm}$ with $100 \mathrm{GHz}$ radiation.

According to Eq. (3), the required Nyquist sampling interval is approximately $1.9 \lambda$. Here, we adopt $2.25 \lambda, 3 \lambda$ and $3.75 \lambda$ sam- 


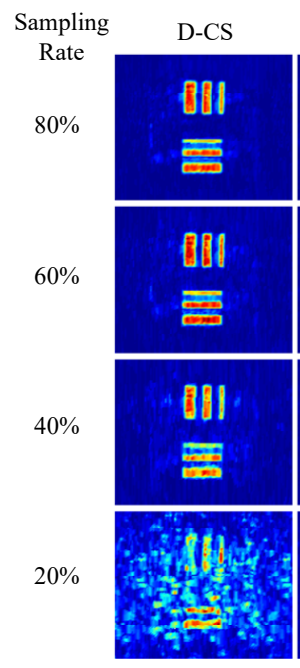

FT-CS

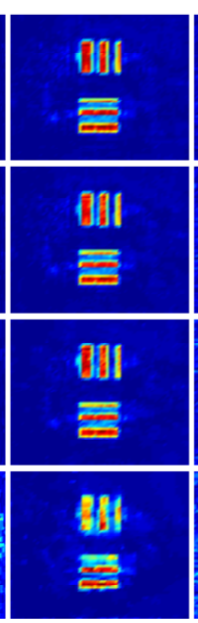

(a)
BP

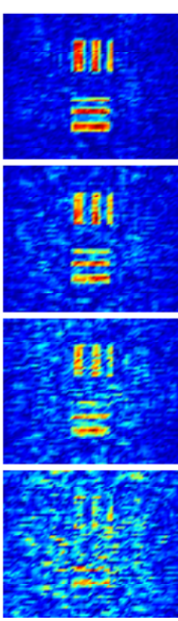

D-CS

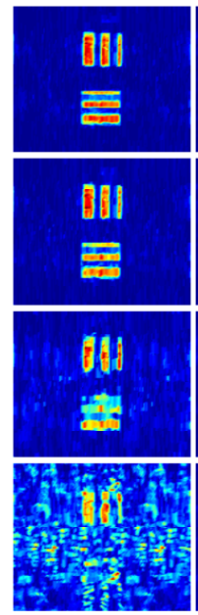

FT-CS

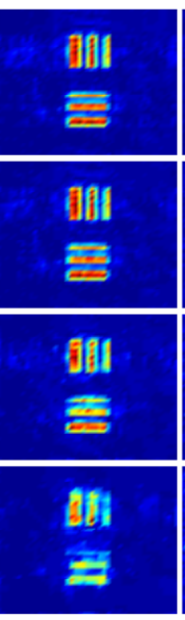

(b)
BP

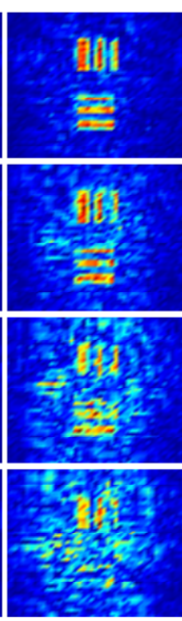

D-CS

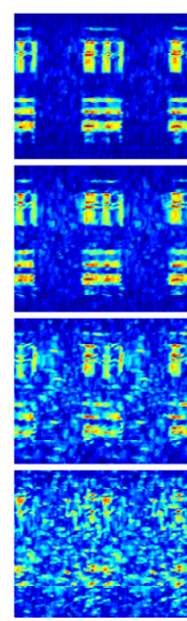

FT-CS

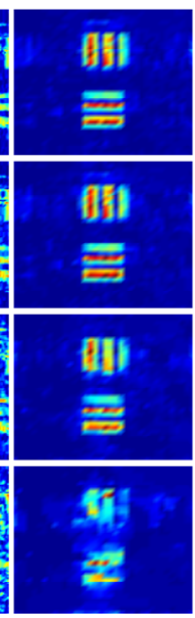

(c)
BP

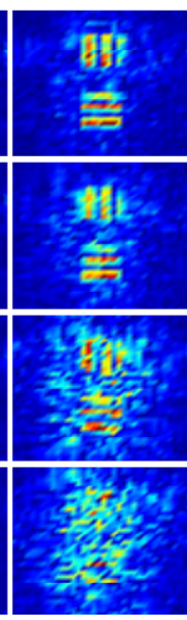

Fig. 11. Experimental results showing the reconstruction quality of three methods based on element spacing of (a) $2.25 \lambda$, (b) $3 \lambda$ and (c) $3.75 \lambda$.

pling interval (element spacing) for comparison. The grid spacing of the target plane is fixed at $\lambda$. Fig. 11 shows the results of D-CS method, FT-CS method and BP method under different sampling rates. Similar to the simulation results, the D-CS method can achieve better resolution than the FT-CS method but has serious ghost image problem in the reconstruction under approximately twice the Nyquist sampling interval. The FT method is more resistant to high random undersampling which can be very useful when fast scanning is needed. Both two CS methods perform better than the BP method with reasonable sampling intervals.

To investigate the robustness of CS methods over different transmitting power, we manually reduce the power by connecting an attenuator between the transmitting antenna and frequency multiplier. The transmitting power is attenuated by 25 $d B, 35 d B, 45 d B$ and $50 d B$. Two random undersampling masks of $100 \%$ sampling rate and $60 \%$ sampling rate are tested with data under $2 \lambda$ uniform sampling interval. From Fig. 12, we can see the number of speckles begin to increase as the attenuation increases. CS based methods show a good noise reduction over BP method. Particularly, the FT-CS method is the most robust to the imaging system with low transmitting power. The comparison between Fig. 12(a) and Fig. 12(b) indicates more measurements can help produce a better image under low transmitting power.

\section{CONCLUSION}

In this paper, CS based holographic imaging techniques have been thoroughly studied. The D-CS method for 2-D planar scanning imaging system has been derived and compared with the existing FT-CS method and the conventional BP method. For the optimal reconstruction of CS methods, sparsity constraint and regularization parameter should be carefully calibrated with respect to different undersampling rates and targets. When the data is randomly undersampled to save scanning time, CS based methods outperforms BP method with better image reconstruction. Particularly, for practical antenna array imaging systems where element spacing does not satisfy Nyquist criterion, D-CS method achieves better resolution than other methods at the cost of much higher computational complexity. However, it should be noted that D-CS method starts to show ghost targets in reconstruction when the element spacing is too large (greater than twice the Nyquist sampling interval in our simulation and experiment). On the other hand, the FT-CS method are more stable than the D-CS method in the case of large sampling interval, low number of measurements and low transmit power. Therefore, for a practical array imaging system, FT-CS method and D-CS method are complimentary techniques and can be used either together or separately depending upon the situation. For example, the FT-CS method can be used to significantly reduce number of samples for a fast but coarse view of the scene. The D-CS method can be later used for higher resolution in the area of interest.

The authors would like to thank Dr. Massimo Candotti for his assistance in the experimental setup.

\section{REFERENCES}

1. R. Appleby and H. Wallace, "Standoff detection of weapons and contraband in the $100 \mathrm{ghz}$ to 1 thz region," Antennas and Propagation, IEEE Transactions on 55, 2944-2956 (2007).

2. D. Sheen, D. McMakin, T. Hall, and R. Severtsen, "Active millimeterwave standoff and portal imaging techniques for personnel screening," in "Technologies for Homeland Security, 2009. HST '09. IEEE Conference on," (2009), pp. 440-447.

3. L. Zhang, Y. Hao, and C. Parini, "Millimetre wave imaging system parameters at $95 \mathrm{ghz}$," Microwaves, Antennas Propagation, IET 5, 528534 (2011).

4. C.-M. Tsai and D. G. Nishimura, "Reduced aliasing artifacts using variable-density k-space sampling trajectories," Magnetic resonance in medicine 43, 452-458 (2000).

5. D. Donoho, "Compressed sensing," Information Theory, IEEE Transactions on 52, 1289-1306 (2006).

6. E. Candes, J. Romberg, and T. Tao, "Robust uncertainty principles: exact signal reconstruction from highly incomplete frequency information," Information Theory, IEEE Transactions on 52, 489-509 (2006).

7. W. L. Chan, K. Charan, D. Takhar, K. F. Kelly, R. G. Baraniuk, and D. M. Mittleman, "A single-pixel terahertz imaging system based on compressed sensing," Applied Physics Letters 93, 121105 (2008). 

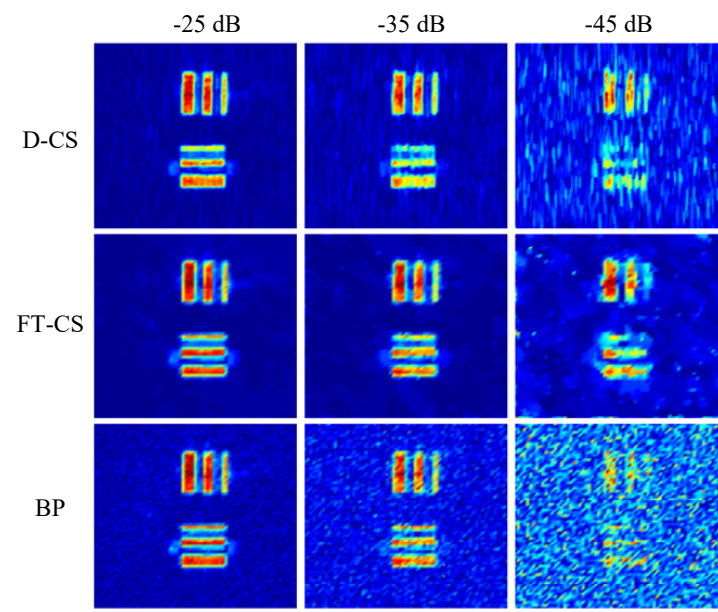

(a)
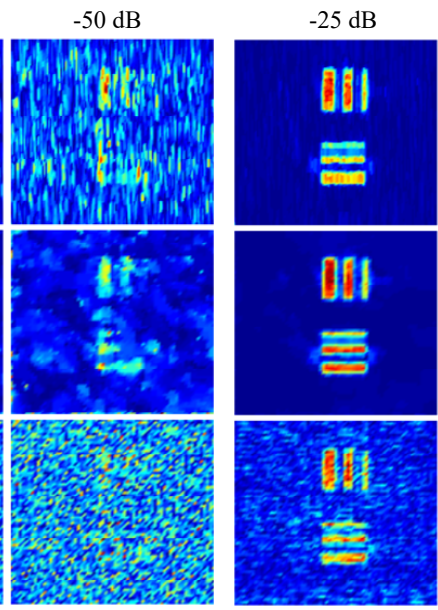
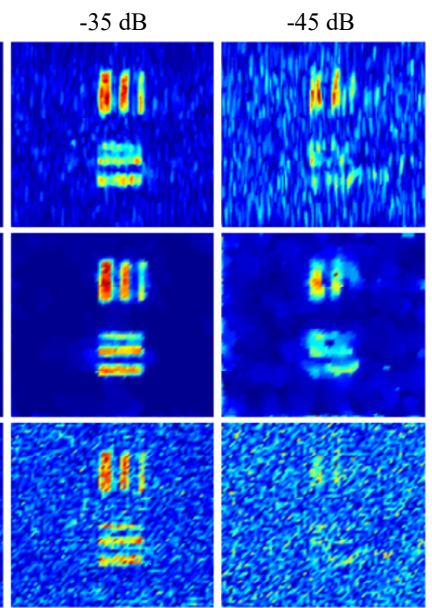

$-50 \mathrm{~dB}$

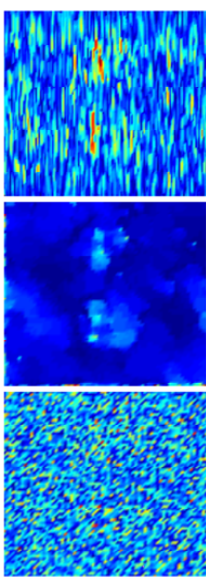

(b)

Fig. 12. Experimental results showing the effect of transmitting power of three methods with different sampling rates. (a) $100 \%$ sampling rate (full sampling). (b) $60 \%$ sampling rate.

8. W. L. Chan, M. L. Moravec, R. G. Baraniuk, and D. M. Mittleman, "Terahertz imaging with compressed sensing and phase retrieval," Optics letters 33, 974-976 (2008).

9. W. L. Chan, H.-T. Chen, A. J. Taylor, I. Brener, M. J. Cich, and D. M. Mittleman, "A spatial light modulator for terahertz beams," Applied Physics Letters 94, 213511 (2009).

10. L. Spinoulas, J. Qi, A. K. Katsaggelos, T. W. Elmer, N. Gopalsami, and A. C. Raptis, "Optimized compressive sampling for passive millimeterwave imaging," Appl. Opt. 51, 6335-6342 (2012).

11. V. M. Patel and J. N. Mait, "Compressive passive millimeter wave imaging with extended depth of field," Optical Engineering 51, 091610-1091610-7 (2012).

12. C. F. Cull, D. A. Wikner, J. N. Mait, M. Mattheiss, and D. J. Brady, "Millimeter-wave compressive holography," Appl. Opt. 49, E67-E82 (2010).

13. L. Qiao, Y. Wang, Z. Shen, Z. Zhao, and Z. Chen, "Compressive sensing for direct millimeter-wave holographic imaging," Appl. Opt. 54, 3280-3289 (2015).

14. R. Baraniuk and $P$. Steeghs, "Compressive radar imaging," in "Radar Conference, 2007 IEEE," (2007), pp. 128-133.

15. A. Gurbuz, J. McClellan, and W. Scott, "A compressive sensing data acquisition and imaging method for stepped frequency gprs," Signal Processing, IEEE Transactions on 57, 2640-2650 (2009).

16. V. Patel, G. Easley, J. Healy, D.M., and R. Chellappa, "Compressed synthetic aperture radar," Selected Topics in Signal Processing, IEEE Journal of 4, 244-254 (2010)

17. J. Yang, J. Thompson, X. Huang, T. Jin, and Z. Zhou, "Randomfrequency sar imaging based on compressed sensing," Geoscience and Remote Sensing, IEEE Transactions on 51, 983-994 (2013).

18. W. Quu, J. Zhou, H. Zhao, and Q. Fu, "Three-dimensional sparse turntable microwave imaging based on compressive sensing," Geoscience and Remote Sensing Letters, IEEE 12, 826-830 (2015).

19. M. Duarte and R. Baraniuk, "Kronecker compressive sensing," Image Processing, IEEE Transactions on 21, 494-504 (2012).

20. Z. Yang and $Y$. Zheng, "Near-field 3-d synthetic aperture radar imaging via compressed sensing," in "Acoustics, Speech and Signal Processing (ICASSP), 2012 IEEE International Conference on," (2012), pp. 2513-2516.

21. H. Kajbaf, J. T. Case, Z. Yang, and Y. R. Zheng, "Compressed sensing for sar-based wideband three-dimensional microwave imaging system using non-uniform fast fourier transform," IET Radar, Sonar \& Navigation 7, 658-670 (2013).

22. D. Liu and P. Boufounos, "Compressive sensing based 3d sar imaging with multi-prf baselines," in "Geoscience and Remote Sensing Sympo- sium (IGARSS), 2014 IEEE International," (2014), pp. 1301-1304.

23. M. Lustig, D. Donoho, and J. M. Pauly, "Sparse mri: The application of compressed sensing for rapid mr imaging," Magnetic resonance in medicine 58, 1182-1195 (2007).

24. J. Haldar, D. Hernando, and Z.-P. Liang, "Compressed-sensing mri with random encoding," Medical Imaging, IEEE Transactions on $\mathbf{3 0}$ 893-903 (2011).

25. L. Carin, D. Liu, W. Lin, and B. Guo, "Compressive sensing for multi-static scattering analysis," Journal of Computational Physics 228, 3464-3477 (2009).

26. L. Poli, G. Oliveri, P. Rocca, and A. Massa, "Bayesian compressive sensing approaches for the reconstruction of two-dimensional sparse scatterers under te illuminations," Geoscience and Remote Sensing, IEEE Transactions on 51, 2920-2936 (2013).

27. G. Oliveri, N. Anselmi, and A. Massa, "Compressive sensing imaging of non-sparse $2 \mathrm{~d}$ scatterers by a total-variation approach within the born approximation," Antennas and Propagation, IEEE Transactions on 62, 5157-5170 (2014).

28. S. Li, G. Zhao, H. Li, B. Ren, W. Hu, Y. Liu, W. Yu, and H. Sun, "Nearfield radar imaging via compressive sensing," Antennas and Propagation, IEEE Transactions on 63, 828-833 (2015).

29. S. Ahmed, A. Schiessl, and L. Schmidt, "A novel fully electronic active real-time imager based on a planar multistatic sparse array," Microwave Theory and Techniques, IEEE Transactions on 59, 35673576 (2011).

30. S. Ahmed, A. Genghammer, A. Schiessl, and L.-P. Schmidt, "Fully electronic $e$-band personnel imager of $2 \mathrm{~m}^{2}$ aperture based on a multistatic architecture," Microwave Theory and Techniques, IEEE Transactions on 61, 651-657 (2013).

31. A. Boyer, P. Hirsch, J. Jordan Jr, L. Lesem, and D. Van Rooy, "Reconstruction of ultrasonic images by backward propagation," in "Acoustical holography," (Springer, 1971), pp. 333-348.

32. J. W. Goodman, Introduction to Fourier optics (Roberts and Company Publishers, 2005)

33. R. Stolt, "Migration by fourier transform," Geophysics $43,23-48$ (1978).

34. M. Soumekh, Synthetic aperture radar signal processing (New York: Wiley, 1999).

35. L. Li, W. Zhang, and F. Li, "Derivation and discussion of the sar migration algorithm within inverse scattering problem: Theoretical analysis," Geoscience and Remote Sensing, IEEE Transactions on 48, 415-422 (2010).

36. J. Case, M. Ghasr, and R. Zoughi, "Optimum two-dimensional uniform spatial sampling for microwave sar-based nde imaging systems," 
Instrumentation and Measurement, IEEE Transactions on 60, 38063815 (2011).

37. D. Sheen, D. McMakin, and T. Hall, "Three-dimensional millimeterwave imaging for concealed weapon detection," Microwave Theory and Techniques, IEEE Transactions on 49, 1581-1592 (2001).

38. Y. Rivenson and A. Stern, "Compressed imaging with a separable sensing operator," Signal Processing Letters, IEEE 16, 449-452 (2009).

39. D. L. Donoho and M. Elad, "Optimally sparse representation in general (nonorthogonal) dictionaries via $\ell_{1}$ minimization," Proceedings of the National Academy of Sciences 100, 2197-2202 (2003).

40. J. Tropp and A. Gilbert, "Signal recovery from random measurements via orthogonal matching pursuit," Information Theory, IEEE Transactions on 53, 4655-4666 (2007).

41. J. M. Bioucas-Dias and M. A. Figueiredo, "A new twist: two-step iterative shrinkage/thresholding algorithms for image restoration," Image Processing, IEEE Transactions on 16, 2992-3004 (2007).

42. S. S. Chen, D. L. Donoho, and M. A. Saunders, "Atomic decomposition by basis pursuit," SIAM journal on scientific computing 20, 33-61 (1998).

43. L. I. Rudin, S. Osher, and E. Fatemi, "Nonlinear total variation based noise removal algorithms," Physica D: Nonlinear Phenomena 60, 259268 (1992).

44. M. Cetin and W. Karl, "Feature-enhanced synthetic aperture radar image formation based on nonquadratic regularization," Image Processing, IEEE Transactions on 10, 623-631 (2001).

45. Z. Xu and E. Y. Lam, "Image reconstruction using spectroscopic and hyperspectral information for compressive terahertz imaging," J. Opt. Soc. Am. A 27, 1638-1646 (2010).

46. J. Trzasko and A. Manduca, "Highly undersampled magnetic resonance image reconstruction via homotopic $\ell_{0}$-minimization," Medical Imaging, IEEE Transactions on 28, 106-121 (2009).

47. "Em software \& systems-s.a. feko." .

48. R. K. Amineh, M. Ravan, A. Trehan, and N. K. Nikolova, "Near-field microwave imaging based on aperture raster scanning with tem horn antennas," Antennas and Propagation, IEEE Transactions on 59, 928940 (2011).

49. Y. Chi, L. Scharf, A. Pezeshki, and A. Calderbank, "Sensitivity to basis mismatch in compressed sensing," Signal Processing, IEEE Transactions on 59, 2182-2195 (2011).

50. T. Zeng, R. Wang, and F. Li, "Sar image autofocus utilizing minimumentropy criterion," Geoscience and Remote Sensing Letters, IEEE 10, 1552-1556 (2013). 